\title{
Responding to adverse patient safety events in Viet Nam
}

\author{
Reema Harrison ${ }^{1 *}\left(\mathbb{D}\right.$, Anurag Sharma ${ }^{1}$, Merrilyn Walton $^{2}$, Esmond Esguerra ${ }^{3}$, Seinyenede Onobrakpor ${ }^{1}$, \\ Bui Trung Nghia ${ }^{4}$ and Nguyen Duc Chinh ${ }^{4}$
}

\begin{abstract}
Background: The psychological and professional impact of adverse events on doctors and nurses is wellestablished, but limited data has emerged from low- and middle-income. This article reports the experiences of being involved in a patient safety event, incident reporting and organisational support available to assist health professionals in Viet Nam to learn and recover.

Method: Doctors and nurses (1000) from all departments of a 1500-bed surgical and trauma hospital in Viet Nam were invited to take part in a cross-sectional survey. The survey explored respondents' involvement in adverse events and/or near miss, their emotional, behavioural and coping responses, experiences of organisational incident reporting, and the learning and/or other consequences of the event. Survey items also assessed the availability of organisational support including peer support and mentorship.

Results: Of the 497 respondents, 295 (59\%) experienced an adverse event in which a patient was harmed, of which $86(17 \%)$ resulted in serious patient harm. 397 (80\%) of respondents experienced a near miss, with 140 of these (28\%) having potential for serious harm. 386 (77\%) reporting they had been affected professionally or personally in some way, with impacts to psychological health $(416 ; 84 \%)$, physical health $(388 ; 78 \%)$, job satisfaction $(378 ; 76 \%)$ and confidence in their ability $(276 ; 56 \%)$ commonly reported. Many respondents were unable to identify local improvements $(373 ; 75 \%)$ or organisation-wide improvements following safety events (359; 72\%) and 171 (34\%) admitted that they had not reported an event to their organisation or manager that they should have.

Conclusions: Health professionals in Viet Nam report impacts to psychological and physical health as a result of involvement in safety events that reflect those of health professionals internationally. Reports of limited organisational learning and improvement following safety events suggest that patient safety culture is underdeveloped in Viet Nam currently. In order to progress work on patient safety cultures and incident reporting in Viet Nam, health professionals will need to be convinced not only that they will not be exposed to punitive action, but that learning and positive changes will occur as a result of reporting safety events.
\end{abstract}

Keywords: Adverse events, Incident reporting, Health policy, Hospitals, Patient safety, Survey research

\section{Background}

An adverse event in healthcare is described as "an injury related to medical management, in contrast to complications of disease) [1]. Around $10 \%$ of patients in developed countries will experience preventable harm during their course of the medical care, with higher estimates obtained from low- and middle-

\footnotetext{
* Correspondence: reema.harrison@unsw.edu.au

${ }^{1}$ Faculty of Medicine, School of Public Health and Community Medicine, UNSW Sydney, Room 308, Samuels Building (F25), Sydney NSW 2052, Australia

Full list of author information is available at the end of the article
}

income settings [2, 3]. Over the past 30 years, many governments and health systems have invested in resources to reduce preventable harm to patients, with incident reporting and retrospective analysis of adverse and near-miss events being a core component of this work [4, 5]. Health systems that measure and report incidents serve an important role in raising awareness of the potential for errors as well as promoting safety cultures [6]. Incident reporting is not a tool that directly remedies problems arising in care; rather it provides a surveillance process that enables

(c) The Author(s). 2019 Open Access This article is distributed under the terms of the Creative Commons Attribution 4.0 International License (http://creativecommons.org/licenses/by/4.0/), which permits unrestricted use, distribution, and reproduction in any medium, provided you give appropriate credit to the original author(s) and the source, provide a link to the Creative Commons license, and indicate if changes were made. The Creative Commons Public Domain Dedication waiver (http://creativecommons.org/publicdomain/zero/1.0/) applies to the data made available in this article, unless otherwise stated. 
exploration and assessment of the risks posed to patients and staff. Reporting, therefore continues to be a foundational process underpinning the development of a patient safety culture. Retrospective analysis of system vulnerabilities is a valuable method, with incident reporting policies and tools embedded in many healthcare system, particularly in high income countries $[5,7,8]$. Reporting plays a role in promoting resilient systems, alongside proactive attempts to optimise the provision of care [9].

Patient safety research has gained momentum over the past 30 years, building knowledge of the key patient safety concerns arising in many developed countries. Less is known about patient safety in low-resource settings. Some low- and middle-income countries are proactively incorporating patient safety goals into national policies, although many countries have yet to collect incident reports at a national level [10-12]. In 2013, the Ministry of Health in Viet Nam mandated a national policy of incident reporting for hospitals. However, since the policy directive there is little evidence of incident reporting systems in hospitals, or where they exist, of being utilised to facilitate learning.

A key feature of organisations using incident reporting is that their health professionals are supported by their organisation; that they feel free to discuss and learn from errors and that a just culture exists [13]. Evidence to date shows that clinicians who directly or indirectly contribute to an adverse event can experience psychological effects that disrupt their professional and personal lives and their ability to deliver high quality, safe care [14-18]. Anxiety, depression, sleep disturbance, fear and worry are consistently reported as well as shame, guilt, loss of self-confidence and feelings of incompetence and worthlessness [1922]. The severity of these effects is often related to the degree of harm to the patient; they are more pronounced with more serious incidents [19, 22].

The detrimental effects following an incident can further harm patients, clinicians, and the wider healthcare system; they can also have flow on effects such as a reduced likelihood of open and honest reporting and discussion of mistakes and adverse events [23]. Safety-conscious industries such as aviation recognize that front line staff will only speak up if they feel supported, have confidence that they will be treated fairly, and that their reports will be used for learning rather than punishment [13]. Until this study, the experiences of health professionals in Viet Nam regarding their experiences of adverse events, of incident reporting or of the organisational support available for health professionals involved in safety events to learn and recover has been unknown. This study therefore aimed to contribute to understanding of these phenomena, and to also explore wider implications for the development of safety culture in Viet Nam.

\section{Methods}

\section{Sample and setting}

Doctors and nurses from all departments of a 1500-bed surgical and trauma and teaching hospital in Viet Nam were invited to participate in the study, with 1000 potential participants invited in total. The study site is a leading surgical hospital for the treatment of injuries, with most patients experiencing trauma in rural and urban regions transferred to the hospital for treatment. The study site operates a system of incident report from doctors, nurses and other staff via the Nursing Department. Anonymous daily and monthly reports are processed through the Quality Control Department. The Quality Control Department staff, with the assistance of the General Planning Department, perform analyses to identify actual or potential medical errors then undertake root -cause analyses of these events. All the reports and analysis are sent to the Directorial Board or presented at weekly meetings in the hospital with the Directors and representatives of all departments present in order to identify solutions and develop interventions where required.

\section{Survey tool}

This survey tool used is a validated instrument previously used with United Kingdom (UK), United States of America (US) and Australasian health professionals, but adapted and translated for use with a Vietnamese sample $[24,25]$. Standard definitions were used to explain the terms 'adverse events' and 'near misses'. Data were collected regarding respondents' involvement in adverse events and/or near miss, their emotional, behavioural and coping responses, experiences of organisational incident reporting, and the learning and/or other consequences of the event. Survey items also assessed the availability of organisational support including mentorship. The translated survey was independently checked by the Vietnamese bilingual research team members to ensure accuracy.

\section{Procedure}

The paper-based survey was administered to doctors and nurses across all departments of an urban surgical hospital in Viet Nam. The Vietnamese research assistant delivered survey packs containing an invitation letter and the survey to doctors and nurses in each department of the hospital and then collected the anonymous, completed surveys one week later. No identifiable information was collected.

\section{Analysis}

The outcome variables of interest were derived from responses to the questionnaire items including involvement in an incident and a range of associated personal and professional outcomes (Fig. 1). Involvement in a 
Adverse

Event Definition: An adverse patient safety event is when something goes wrong in the patient's care that could cause them harm and that could have been prevented.

Section 1: Demographic information

a) Age, sex, time in role.

Section 2: Safety event details, personal and professional effects

a) Based on the above definitions, do you believe you have ever been involved any of the following in your career? (Please tick all that apply) Adverse event with serious patient harm; Adverse event with minor patient harm; Near miss with potential for serious patient harm; Near miss with potential for minor patient harm

b) Do you believe that involvement in a near miss or adverse event has affected your professional or personal life for any period of time? $(\mathrm{Y} / \mathrm{N})$

c) Do you believe that involvement in an adverse event has affected your: $(\mathrm{Y} / \mathrm{N}) \mathrm{Job}$ satisfaction; Confidence in your ability as a doctor; Professional reputation; Physical health and wellbeing; Mental health and wellbeing; Ability to sleep; Relationship with colleagues

d) Please indicate your extent of agreement with each of the statements below (1 Strongly disagree -5 Strongly agree): Being involved in an adverse event has made me feel anxious about the potential for future errors; generally distressed (e.g. depressed, upset, angry); generally anxious (e.g. nervous, panicky, tense); generally bad about myself (e.g. shame, guilt, feeling incompetent); more confident in my abilities (e.g. effective, competent); determined to improve my practice (e.g. determined, strong)

Section 3: Support after safety events

a) Who did you talk to about the adverse event? (tick all that apply) Colleague (peer); Colleague (senior); Colleague (other profession); Family/friends; Manager; Supervisor; Patient/carer; No-one; Healthcare provider (eg own GP); Other (specify)

b) Do you agree with the following statement?: $(\mathrm{Y} / \mathrm{N})$ "My organisation adequately support doctors and nurses who have been involved in adverse events"

Section 4: Reporting and disclosure of events

a) Have you ever disclosed a: $(\mathrm{Y} / \mathrm{N})$. Serious adverse event to a patient/carer; Minor adverse event to a patient/carer

b) Have you ever reported an adverse event to your manager or supervisor? $(\mathrm{Y} / \mathrm{N})$

c) IF YES: How happy were you with how your manager or supervisor responded? (1 very unhappy to 5 very happy)

d) Have you ever been involved in an adverse event that you felt should be reported to the managers but that you did not report? $(\mathrm{Y} / \mathrm{N})$

Section 5: Experiences after reporting a safety event

a) Did you experience any of the following after reporting an event to you manager or supervisor?: $(\mathrm{Y} / \mathrm{N})$

b) Being blamed; Changes in protocols or guidelines; Changes in the way you work in your department; Useful guidance for future practice; Support from colleagues; More supervision; Punishment; More training

c) Have you ever supported another colleague who has been involved in an adverse event? $(\mathrm{Y} / \mathrm{N})$

d) How confident do you feel about supporting a colleague who has been involved in an adverse event? ( 1 not at all to 5 very confident)

Section 6: Option for open text comments regarding organisational support and overall experiences.

Fig. 1 Summary of survey components 
near miss or adverse event was captured through four response options: i) adverse event with serious patient harm, ii) adverse event with minor patient harm, iii) near miss with potential for serious patient harm, iv) near miss with potential for minor patient harm or v) none of these. Descriptive statistical analysis was undertaken using the Stata 15 package.

\section{Results}

Of the 1000 invited participants, 497 responded (50\% response rate). Table 1 displays the demographic data relating to the sample. Most respondents were aged 2544 years $(403 ; 81 \%)$ and mid-career $(273 ; 55 \%)$, with a slightly stronger representation of female respondents (300; 60\%). A cross-section of specialty backgrounds was apparent, with anesthesia $(119 ; 24 \%)$, orthopedics $(69$; $14 \%)$ and emergency care $(58 ; 12 \%)$ as the largest specialty groups, reflecting the status of this hospital as a trauma and surgical centre.

Table 2 outlines the nature of adverse events and near miss events experienced by the respondents. Just over half experienced an event harming a patient, 295 (59\%) of which 86 (17\%) experienced an event resulting in serious patient harm. Most respondents reported experiencing a near miss $(397 ; 80 \%)$, with 140 of these $(28 \%)$ having potential for serious harm. Many respondents had disclosed their adverse event $(172 ; 35 \%)$ or near miss $(233 ; 47 \%)$ to a

Table 1 Descriptive Statistics: Demographics

\begin{tabular}{lll}
\hline Covariates & $N$ & $\%$ \\
\hline Experience (Years in Practice) & 84 & 16.99 \\
$\quad$ <2 Years & 273 & 54.96 \\
2-10 Years & 140 & 28.17 \\
$>$ 10 Years & & \\
Age Categories (\%) & 55 & 11.07 \\
$\quad$ <25 years & 296 & 59.56 \\
25-34 years & 107 & 21.53 \\
35-44 years & 32 & 6.44 \\
45-54 years & 7 & 1.41 \\
55-64 years & & \\
Gender (\%) & 197 & 39.64 \\
Male & 300 & 60.36 \\
Female & & \\
Speciality (\%) & 119 & 23.94 \\
Anaesthesia & 69 & 13.88 \\
Orthopaedic & 58 & 11.67 \\
ED & 32 & 6.43 \\
Spinal & 187 & 6.44 \\
Surgery & & 37.64 \\
Other & 52 & \\
\hline
\end{tabular}

Number of Observations (N): 497
Table 2 Descriptive Statistics: Outcome Variables

\begin{tabular}{ll}
\hline Incident type & N \\
\hline Serious Harm & 86 \\
Actual & 140 \\
Near miss & \\
Minor Harm & 209 \\
Actual & 257 \\
Near miss & \\
Incident Reporting & 233 \\
Disclosed minor event to patient/carer & 172 \\
Disclosed serious event to patient/carer & 407 \\
Reported near miss/serious event to manager & 171 \\
Not reported an event that you should have
\end{tabular}

Number of Observations (N): 497

patient or carer. Most respondents had reported an event to a manager (407; 82\%), but 171 (34\%) admitted that there had been events that they had not reported to their organisation or manager when they should have.

Table 3 shows little learning and change occurred after reporting an incident. All respondents reported 'slightly' or 'not at all' when answering this question despite the five-point response scale provided. Most respondents said they received little useful feedback or empathy from their colleagues $(426 ; 86 \%)$, with many unable to identify local improvements $(373 ; 75 \%)$ or organisation-wide improvements such as changes to protocols $(359 ; 72 \%)$. Respondents reported not being blamed (341;67\%), subject to closer supervision (380; 76\%) or punished (332; 67\%).

The personal and professionals impacts of the event on respondents were apparent (see Table 4), with 386 (77\%) reporting they had been affected professionally or personally in some way. A significant majority reported the event had impacted their mental health (416; 84\%), substantial numbers of respondents said their physical health had also been affected (388; 78\%). The professional implications of being involved in an event were demonstrated in impacts on job satisfaction (378; 76\%) and their confidence in the ability (276; 56\%).

As shown in Table 5, respondents commonly sought the support of peers at their own rank or level (338; $68 \%)$ and/or at a senior rank or level to them $(266 ; 45 \%)$ as a result of their involvement in an event. Managers (219; 44\%) and/or supervisors $(98 ; 20 \%)$ were also commonly referred to for support, with family and friends $(155 ; 31)$ also valued. Smaller groups of respondents sought support from a mentor $(98 ; 20 \%)$ or referred to their own healthcare provider (108; 22\%). Many respondents indicated they felt that their organisation offered adequate support to doctors and nurses involved in safety events $(217 ; 43.66 \%)$. There was a significant correlation between those who did not perceive the support 
Table 3 Outcomes of incident reporting

\begin{tabular}{|c|c|c|}
\hline Outcomes & $\mathrm{N}$ & $\%$ \\
\hline \multicolumn{3}{|l|}{ Blamed } \\
\hline Not at all & 341 & 68.61 \\
\hline Slightly & 120 & 24.15 \\
\hline Missing/No response & 36 & 7.24 \\
\hline \multicolumn{3}{|c|}{ Changed Protocol Guidelines } \\
\hline Not at all & 359 & 72.23 \\
\hline Slightly & 95 & 19.11 \\
\hline Missing/No response & 43 & 8.66 \\
\hline \multicolumn{3}{|l|}{ Changed Local level } \\
\hline Not at all & 373 & 75.05 \\
\hline Slightly & 86 & 17.3 \\
\hline Missing/No response & 38 & 7.65 \\
\hline \multicolumn{3}{|l|}{ Useful Feedback } \\
\hline Not at all & 426 & 85.71 \\
\hline Slightly & 35 & 7.04 \\
\hline Missing/No response & 36 & 7.25 \\
\hline \multicolumn{3}{|l|}{ Colleague Empathy } \\
\hline Not at all & 426 & 85.71 \\
\hline Slightly & 36 & 7.24 \\
\hline Missing/No response & 35 & 7.05 \\
\hline \multicolumn{3}{|l|}{ Closer Supervision } \\
\hline Not at all & 380 & 76.46 \\
\hline Slightly & 77 & 15.49 \\
\hline Missing/No response & 40 & 8.05 \\
\hline \multicolumn{3}{|l|}{ Punishment } \\
\hline Not at all & 332 & 66.8 \\
\hline Slightly & 128 & 25.75 \\
\hline Missing/No response & 37 & 7.45 \\
\hline
\end{tabular}

offered by the organisation was adequate and being involved in an event with serious patient harm $(0.14$, p- value 0.001).

\section{Discussion}

Our findings demonstrate significant personal and professional impact on doctors and nurses who are involved

Table 4 Personal Effect of involvement in Adverse Event

\begin{tabular}{lll}
\hline Covariates & N & $\%$ \\
\hline Affected in general & 386 & 77.67 \\
Affected job satisfaction & 378 & 76.06 \\
Affected confidence in skills & 268 & 53.92 \\
Affected physical health & 388 & 78.07 \\
Affected mental health & 416 & 83.70 \\
\hline
\end{tabular}

Number of Observations (N): 497 in AEs and near misses in the study hospital, which reflects international evidence $[14,15]$. The pervasive impact of $\mathrm{AE}$ and error involvement was evident through the physical and mental health impacts, in addition to reducing job satisfaction. When compared to findings using the same survey instrument in the UK and in Australasia, the proportion of the Vietnamese sample reporting detrimental effects to confidence in their skills and mental health was similar to that reported in Australasia and in the UK. Detriment to physical health and job satisfaction however was higher than in studies in the UK and Australasia [24, 25]. A substantial group of respondents (34\%) identified that they had not reported events that they should have, which compares with $25 \%$ of the UK and Australasian samples [24, 25].

Our data contribute to a growing body of work internationally, predominantly spanning the US, Canada and Europe, that has explored the 'second victim' phenomena of health professional impacts from involvement in safety events [26-29]. Data from our study sample in Viet Nam suggests that, unlike most developed country contexts, the majority of health professionals would talk to a manager and also to patients about safety events. These findings indicate that contextual factors relating to health system such as consumer empowerment, litigious culture, and organisational hierarchy, may influence actions that follow safety events [30]. In addition, as a trauma referral centre in high demand, health professionals in the study hospital have power in their relationship with patient, see large patient numbers and generally have less ongoing contact with them. Such factors may contribute to explaining the high proportion of disclosures made to patients.

The relevant absence of person-level, local-level or organisational-level actions resulting from a safety event emerged strongly in our data. When asked about experiences following a safety event, it was clear that, whilst respondents mostly did not report feeling blamed or punitive action, there was very little in the way of local or organisational improvement. The consistency of 'not at all' or 'slightly' responses to this group of items suggests that actions following a safety events are either rarely taken across the organisation or possibly that respondents did not wish to report these actions. A result of these data was that the degree to which organisational learning may occur as a result of a safety incident was unclear.

In keeping with the international evidence regarding clinician response to $\mathrm{AE}$ and error involvement, peers were identified as the most popular and valued source of support [31-33]. But unlike in the data emerging from developed countries, managers were equally identified as important sources of support. Conversely, discussions with patients and family members following an event were reported by less than half of the respondents in the 
Table 5 Sources of support post serious and minor adverse events/near misses

\begin{tabular}{ll}
\hline Variable & $\mathrm{N}$ \\
\hline Peer & 338 \\
Senior Peer & 266 \\
Colleague from other professions & 121 \\
Friends and Family & 155 \\
Manager & 219 \\
Mentor & 98 \\
Patient & 79 \\
No one & 32 \\
Own Healthcare Provider & 108 \\
\hline
\end{tabular}

Number of Observations (N): 497

present study, particularly in the context of serious events $(35 \%)$ that would be associated with incident disclosure processes in many developed countries [34, 35]. Organisational support was identified as important in the context of AE and error involvement. Those who did not perceive the support offered by the organisation was adequate were significantly more likely to be those who were involved in an event with serious patient harm. As cross-sectional data, it is not possible to draw conclusive statements regarding the nature of this relationship but evidence in the research literature to date indicates two potential relationships between support and incidence of safety events. There is some evidence to suggest that those are better supported in their organisation, for example through mentorship, may be less likely to be involved in safety events or more serious events [13, 19, 36, 37]. Alternatively, being involved in an event with a harmful or more serious patient outcome may leave health professionals feeling unsupported by their organisation.

\section{Implications}

The study findings have implications for organisational learning in relation to adverse events and errors, for supporting health professionals involved in these events, and for the development of effective incident reporting systems in Vietnamese hospitals. Optimal organisational learning is said to occur when an organisation looks beyond the events and circumstances immediately preceding an incident (single-loop learning) to explore the conditions within the organisation that have enabled the immediate circumstances to arise; characterised as double loop learning [38-40]. Limited evidence of any actions arising in response to an $\mathrm{AE}$ or near miss in the present study suggests that there is currently a lack of learning at a local or organisational level in response to the events occurring. An effective incident reporting system that promotes local and organisational learning activities after events is one mechanism to routinely embed learning as part of an incident management process. Systematic incident reporting and management can facilitate reflection on actions relating to the events arising but also to the latent factors that allowed the event to occur.

Establishing a strong patient safety culture within organisations at a national level is critical for the emergence of an effective incident management process. Fundamental to developing a strong patient safety culture, is the ability to identify and communicate safety concerns or challenges to the senior management of an organisation [13]. In order to progress work on patient safety cultures and incident reporting in Viet Nam, health professionals will need to be convinced not only that they will not be exposed to punitive action, but that learning and positive changes will occur as a result of reporting safety events. In progressing towards a strong safety culture, training of peers and managers (who are identified as the key sources of support following events) around impacts of AEs and errors may also contribute to culture change.

\section{Limitations}

Whilst our findings reflect those of the other crosssectional survey studies on this topic, a cross-sectional method is reliant on retrospective recall and may therefore lead to recollection bias. Cross-sectional selfreporting also limits the accuracy of information gathered regarding the severity and duration of emotions experienced in relation to an adverse event or near miss. However, the exploration of stable beliefs rather than specific experiences is of value in the context of the present study which seeks to know how experiences of involvement in AEs and errors has shaped reporting behavior and the emerging safety culture. The use of a responder sample may have influenced the resulting findings, despite the strong response rate; those who were strongly impacted or not impacted at all by their experiences may have declined to participate. The findings provide valuable insight into an issue that is difficult to discuss, particularly in developing country contexts such as Viet Nam. The management of adverse events and errors has significant implications for both clinician well-being and patient safety.

\section{Conclusion}

Establishing a strong patient safety culture in Vietnamese hospitals is a necessary foundation for effective incident management, and subsequent learning to improve the safety of health care. The ability to identify and communicate safety concerns or challenges to the senior management of an organisation is essential. Work focusing on building a learning and just culture is required. As a first step health professionals need a 
mind-set where they believe that positive change to enhance safety will arise from talking openly about safety events. Healthcare leaders and managers have a critical role in affecting change in their organisations and sharing this knowledge within and between services.

\section{Abbreviations}

AE: Adverse event; UK: United Kingdom; US: United States of America

\section{Acknowledgements}

We would like to acknowledge and thank all of the staff who contributed to the study as participants in the study hospital and those who supported the research process.

\section{Authors' contributions}

$\mathrm{RH}$ and $\mathrm{MW}$ were responsible for the conception of the study and development of survey instrument. $\mathrm{RH}, \mathrm{EE}, \mathrm{NC}$, BT were responsible for recruitment, data collection and preliminary analysis. EE, SO were responsible for data cleaning. $\mathrm{RH}$, SO and AS were responsible for the analytic strategy and analysis undertaken. All authors contributed to the development of the manuscript. All authors reviewed the final manuscript. All authors read and approved the final manuscript.

\section{Funding}

This project was funded by a Cluster Grant from the Sydney South-East Asia Centre. The funding body had no role in in the design of the study and collection, analysis, and interpretation of data or in writing the manuscript.

\section{Availability of data and materials}

Study data is held by the study team and cannot be shared in accordance with ethical approval but may be sought from the lead author.

\section{Ethics approval and consent to participate}

The study received ethics approval from The University of Sydney Human Research Ethics Committee (Project no: 2015/504) and from the Hospital Board. Written consent was obtained from participants.

\section{Consent for publication}

Participants consented to the research findings being submitted for publication and to the publication of these.

\section{Competing interests}

There are no competing interests to declare.

\section{Author details}

'Faculty of Medicine, School of Public Health and Community Medicine, UNSW Sydney, Room 308, Samuels Building (F25), Sydney NSW 2052, Australia. ${ }^{2}$ Faculty of Medicine and Health, School of Public Health, The University of Sydney, Sydney, Australia. ${ }^{3}$ Office for Global Health Faculty of Medicine and Health, The University of Sydney, Sydney, Australia. ${ }^{4}$ Viet Duc University Hospital, Hanoi, Vietnam.

Received: 25 March 2019 Accepted: 9 September 2019 Published online: 18 September 2019

\section{References}

1. Wears RL, Wu AW. Dealing with failure: the aftermath of errors and adverse events. Ann Emerg Med. 2000;39:344-6.

2. de Vries EN, Ramrattan MA, Smorenburg SM, Gouma DJ, Boermeester MA The incidence and nature of in-hospital adverse events: a systematic review. Qual Saf Health Care. 2008;17(3):216-23.

3. Wilson RM, Michel P, Olsen S, et al. Patient safety in developing countries: retrospective estimation of scale and nature of harm to patients in hospital. BMJ. 2012:344:e832.

4. Giles S, Fletcher M, Baker M, Thomson R. Incident reporting and analysis Patient safety: research into practice OUP. 2005:108-7.

5. Stavropoulou C, Doherty C, Tosey P. How effective are incident-reporting systems for improving patient safety? A systematic literature review. The Milbank Quarterly. 2015;93(4):826-66.
6. Vincent C. Incident reporting and patient safety; 2007. p. 51.

7. UK Department of Health. National reporting and learning service. Available at: https://report.nrls.nhs.uk/nrlsreporting/ [accessed 17 Jan 2019].

8. New South Wales Health, 2014. Incident management policy. Available at: https://www1.health.nsw.gov.au/pds/ActivePDSDocuments/PD2014_004.pdf].

9. Braithwaite J, Wears RL, Hollnagel E. Resilient health care: turning patient safety on its head. Int J Qual Health Care. 2015;27(5):418-20.

10. World Health Organisation, 2016 Model for Patient Safety Reporting and Learning Systems. Available from: https://www.who.int/patientsafety/topics/ reporting-learning/mim/user-guide/en [accessed 17 Jan 2019].

11. Wung CH, Yu TH, Shih CL, Lin CC, Liao HH, Chung KP. Is it enough to set national patient safety goals? An empirical evaluation in Taiwan. Int J Qual Health Care. 2011;23(4):420-8.

12. World Health Organisation, 2017. Creating a culture of patient safety in China. Available at: http://www.wpro.who.int/china/mediacentre/releases/2017/201 70213-creating-a-culture-for-patient-safety/en/ [accessed 17 Jan 2019].

13. Dekker S. Just culture: balancing safety and accountability: CRC Press; 2016.

14. Sirriyeh R, Lawton RJ, Gardner P, et al. Coping with medical error: a systematic review of papers to assess the effects of involvement in medical error on health care professional's psychological well-being. Qual Saf Health Care. 2010;19:1-8.

15. Harrison R, Lawton R, Stewart K. Doctors' experiences of adverse events in secondary care: the professional and personal impact. Clinical Medicine. 2014;14:585-90.

16. Lawton RJ, Parker D. Barriers to reporting incidents in a health care system. Qual Saf Health Care. 2002;11:15-8.

17. Kingston MJ, Evans SM, Smith BJ. Et al attitudes of doctors and nurses towards incident reporting: a qualitative analysis. Med J Aust. 2004;18:136-9.

18. Evans SM, Berry JG, Smith BJ, et al. Attitudes and barriers to incident reporting: a collaborative hospital study. Qual Saf Health Care. 2006;15(1):39-43.

19. Seys $D$, Scott $S, W u$ A, et al. Supporting involved health care professionals (second victims) following an adverse health event: a literature review. Int J Nurs Stud. 2013;50(5):678-87.

20. Engel $K G$, Rosenthal $M$, Sutcliffe $K M$. Residents' responses to medical error: coping, learning and change. Acad Med. 2006;81:86-93.

21. Waterman AD, Garbutt J, Hazel E, et al. The emotional impact of medical errors on practicing physicians in the United States and Canada. Jt Comm J Qual Patient Saf. 2007:33:467-76.

22. Scott SD, Hirschinger LE, Cox KR, McCoig M, Brandt J, Hall LW. The natural history of recovery for the healthcare provider "second victim" after adverse patient events. BMJ Quality and Safety. 2009;18(5):325-30.

23. Gallagher $T H$, Waterman AD, Ebers AG, Fraser VJ, Levinson W. Patients' and physicians' attitudes regarding the disclosure of medical errors. Jama. 2003; 289(8):1001-7.

24. Harrison R, Lawton R, Perlo J, et al. Emotion and coping in the aftermath of error: a cross country exploration. J Pat Saf. 2015;11(1):28-35.

25. Harrison $\mathrm{R}$, Lee $\mathrm{H}$, Sharma A. A survey of the impact of patient adverse events and near misses on anaesthetists in Australia and New Zealand. Anaesth Intensive Care. 2018:46(5):510-5.

26. Mira JJ, Carrillo I, Lorenzo S, et al. The aftermath of adverse events in Spanish primary care and hospital health professionals. BMC Health Serv Res. 2015;15(1):151.

27. Ferrús L, Silvestre C, Olivera G, Mira JJ. Qualitative study about the experiences of colleagues of Health professionals involved in an adverse event. Journal of patient safety. 2016

28. Vanhaecht K, Seys D, Schouten L, Bruyneel L, Coeckelberghs E, Panella M, Zeeman G. Duration of second victim symptoms in the aftermath of a patient safety incident and association with the level of patient harm: a cross-sectional study in the Netherlands. BMJ Open. 2019;9(7):e029923.

29. Ullstrom S, Andreen Sachs M, Hansson J, et al. Suffering in silence: a qualitative study of second victims of adverse events. BMJ Qual Saf. 2014 Apr;23(4):325-31.

30. Giroud S, Aro I. Cultural issues in litigation in the Asia Pacific region: myth or reality? In: IBA annual conference 2010: report on the joint session of the IBA litigation committee and the Asia Pacific forum.

31. Hu YY, Fix ML, Hevelone ND, Lipsitz SR, Greenberg CC, Weissman JS, Shapiro J. Physicians' needs in coping with emotional stressors: the case for peer support. Arch Surg. 2012:147(3):212-7.

32. Scott SD, Hirschinger LE, Cox KR, et al. Caring for our own: deploying a systemwide second victim rapid response team. Joint Commission journal on quality and patient safety. 2010;36(5):233-40. 
33. Edrees H, Connors C, Paine L, Norvell M, Taylor H, Wu AW. Implementing the RISE second victim support programme at the Johns Hopkins Hospital: a case study. BMJ Open. 2016;6(9):e011708.

34. Australian commission on safety and quality in Health care, 2013. Open

Disclosure Framework. Available at: https:/www.safetyandquality.gov.au/ourwork/open-disclosure/the-open-disclosure-framework/ [accessed 17 Jan 2019].

35. NHS England. Serious incident Framework. Available at: https://www. england.nhs.uk/wp-content/uploads/2015/04/serious-incidnt-framwrk-upd. pdf [accessed 17 Jan 2019].

36. Clarke SP, Rockett JL, Sloane DM, Aiken LH. Organizational climate, staffing, and safety equipment as predictors of needlestick injuries and near-misses in hospital nurses. Am J Infect Control. 2002;30(4):207-16.

37. Harrison R, Sharma A, Lawton R, Stewart K.. Is physician mentorship associated with the occurrence of adverse patient safety events? J Pat Saf (in press: accepted 15 Jan 2019).

38. Argyris C. Double loop learning in organizations. Harv Bus Rev. 1977;55(5): $115-25$.

39. Reason J. Human error: models and management. BMJ. 2000;320(7237):768-70

40. Edmondson AC. Learning from mistakes is easier said than done: group and organizational influences on the detection and correction of human error. J Appl Behav Sci. 2004;40(1):66-90.

\section{Publisher's Note}

Springer Nature remains neutral with regard to jurisdictional claims in published maps and institutional affiliations.

Ready to submit your research? Choose BMC and benefit from:

- fast, convenient online submission

- thorough peer review by experienced researchers in your field

- rapid publication on acceptance

- support for research data, including large and complex data types

- gold Open Access which fosters wider collaboration and increased citations

- maximum visibility for your research: over $100 \mathrm{M}$ website views per year

At BMC, research is always in progress.

Learn more biomedcentral.com/submissions 